\title{
Intra-subject Variability of Impact Shock of Foot on the Ground during Walking and Running Activities
}

\author{
Graziella Aghilone $^{1}$, Massimo Cavacece ${ }^{2, *}$ \\ ${ }^{1}$ Pharmacy and Medicine Faculty, University La Sapienza of Rome, Via Regina Margherita n.273, 00198 Rome (RM), Italy \\ ${ }^{2}$ Department of Civil and Mechanical Engineering, University of Cassino and Lazio Meridionale, \\ Via G. Di Biasio n.43, 03043 Cassino (FR), Italy.
}

Copyright (C) 2018 by authors, all rights reserved. Authors agree that this article remains permanently open access under the terms of the Creative Commons Attribution License 4.0 International License

\begin{abstract}
Aim: The intra-subject variability is evaluated by a deterministic acceleration model in the frequency content between walking and running activities. The usefulness of this research is to explore the dependence of peak acceleration of foot on different pedestrian's velocity. Method: The mathematical model can be represented in time domain as a sum of Fourier harmonic components. The mathematical approach is applied to fit the accelerations of the foot, acquired during the impact shock of the human body on treadmill during walking and running activities at different speeds. Spectral analysis evaluates the frequency field of impact shock during walking and running activities. Results: The fitting of experimental data, by a mathematical approach, offers the analysis of the peak force of the foot on the ground, the dynamic acceleration factor (DAF) and the activity rate harmonics during walking and running activities. Conclusion: Marked differences in vertical accelerations are illustrated between walking and running activities. Recommendations could be deducted with regard to the dose of impacts that can be beneficial or detrimental to human health.
\end{abstract}

Keywords Spectral Analysis, Impact Shock, Walking/Running Activities

\section{Introduction}

Back-pain disease, Parkinson's disease, obesity, risk of falling, venous insufficiency, walking and running activity have been monitored by accelerometry in clinical and nonclinical-based studies.

Foot switches, gait mats, force plates, optical motion analysis are other techniques to investigate human movement. Respect to previous techniques, the accelerometers represent the best preferred choice to detect human movement during continuous analysis. The reasons of the preferred choice for the accelerometers are the following aspects:
- the accelerometers are characterized by the state of very low power consumption;

- lightweight compact data acquisition instruments;

- off-line and real time data processing techniques.

- the accelerometers represent a non-intrusive means of assessing postures, to assess energy expenditure and estimate physical activity.

Accelerometers can be used to estimate the impact conditions and a more localized effect of the impact of the foot. In marked contrast, the restriction of the use of accelerometers depends on the soft tissue movement, relative to the bone that can influence the resulting peak accelerations.

The measurement of the ground reaction forces could offer another experimental technique to evaluate the impact between foot and ground. Also the measurement of the ground reaction forces is characterized by restrictions. The footground impact is a very short phenomenon. The duration of impact is typically less than $50 \mathrm{~ms}$. During this brief period of time the shoe midsole is compressed, biological materials such as the calcaneal fat pad, ligaments, cartilage and bone are deformed, and the muscle tendon complexes of lower extremity joints are stretched. This is essentially a passive process because the duration is too short to actively respond to the impact. The most direct measure of the impact would be a recording of the forces in the responsive tissues. However, this would involve invasive instrumentation of these tissues. The restriction is that the ground reaction forces are influenced by the entire mass of the body. Therefore every experimental technique presents restrictions.

This study examines the impact of the foot during walking and running activity by the accelerometers. The shock attenuation of the foot is characterized by a combination of active and passive mechanisms.

Passive mechanisms are responsible for attenuating higher frequency components and include deformation of the shoe, heel fat pad, ligaments, bone, articular cartilage, and oscillation of soft tissue compartments [1]. 
The eccentric muscle contractions, increased muscle activation, changes in segment geometry, and adjustments in joint stiffness are active shock attenuation mechanisms. The plantar intrinsic and plantar extrinsic muscle, being under direct central nervous system control, increase or decrease the stiffness of the medial and lateral longitudinal arches of the foot.

The acceleration of foot generates the frequency content and signal power of the impact shock. The foot acceleration profile, in walking and running activities, contains the following ranges: a lower frequency range $(4-8 \mathrm{~Hz})$ representing voluntary lower extremity motion and the vertical acceleration during the stance phase; a higher frequency range (10$20 \mathrm{~Hz}$ ) representing the rapid deceleration of the foot at initial ground contact. This study investigates the frequency range 0-20 Hz.

With reference to the aforementioned frequency ranges, the deformation of the shoe, heel fat pad, ligaments, bone, articular cartilage, and oscillation of soft tissue compartments are passive mechanisms that mitigate higher frequency components. The active mechanisms attenuate lower frequency components of accelerations.

Vaughan et al. [2] analyzed the kinematics and dynamics of joints:

- the angular velocity and angular acceleration of the right calf muscle relative to its vertical axis, evaluated as a function of time.

- the fore-aft, mediolateral, and vertical forces in newtons $(\mathrm{N})$, acting on the subject's right foot, as a function of time.

- the flexion extension moments (in newton-metres) at the right hip, knee, and ankle joints plotted as a function of time.

- the flexion/extension, internal/external rotation, and abduction/ adduction moments in newton-metres, at the right knee joint evaluated as a function of time.

Non-linear responses of the human body exposed to whole-body vibration at different magnitudes have been observed in various studies. Matsumoto and Griffin observed median apparent masses and phases in vertical axis and in the fore-and-aft axis of eight subjects measured with random and sinusoidal vibration at five frequencies $(3.15,4.0,5.0$, 6.3 and $8.0 \mathrm{~Hz})$ frequencies and five magnitudes $(0.35,0.5$, $0.7,1.0$ and 1.4 and $\mathrm{m} / \mathrm{s}^{2}$ r.m.s.) with different postures. [3]. although some subjects showed the expected decrease in the non-linear trend The Authors find that the possible causes of the difference between individuals in the effect of muscle tension on the apparent mass mentioned above could include individual differences in the ability to control particular muscles at a particular tension.

The study of Lim et at. aimed to identify adaptive changes in running kinematics and impact shock transmission as a function of head stability requirements [4]. In more detail, the Authors estimated kinematics and dynamics aspects:
- mean tibia and head acceleration characteristic profile in time and frequency domain during the stance phase as a function of visual;

- mean transfer functions between tibia and head accelerometer signals running at different visual conditions.

A complex mathematical model was proposed by Nigam and Malik. The model was a 15 DOF mass-spring system. The method of evaluation of the stiffness of the spring elements represented the novelty of this study. To evaluate the stiffness of the segment, the Authors considered the axial tension of a truncated ellipsoid. Elastic modulus of the ellipsoidal segment depended on elastic modulus of bone and elastic modulus of tissue. The limit of this study was that the model did not include any damping capability [5].

The greatest portion of the power in the spectrum of the shank signal was found to reside below $20 \mathrm{~Hz}$, in two characteristic peaks. The first peak occurred in the $4-8 \mathrm{~Hz}$ range and was associated with the low frequency motion of the leg during the contact phase. The second peak, associated with the higher frequency, higher amplitude impact shock wave, was found at $12-20 \mathrm{~Hz}$. These frequency domain peaks correspond to the active and impact phases of the time-domain ground reaction force [7].

This brief examination confirmes the requirement to expand the knowledge about impact shock during walking and running activities on the ground.

The purpose of this study is to evaluate the intra-subject variability in the frequency content between walking and running activities. It is proposed a deterministic acceleration model. This approach takes no account of random variation among human population. Therefore, we suggest a uniform acceleration model for individual pedestrian. The mathematical model can be represented in time domain as a sum of Fourier harmonic components. The mathematical model is optimized according to minimum value of sum of squares due to error between numerical results, obtained by mathematical model, and experimental data.

The experimental data were the accelerations of the foot due to the impact shock of the human body on treadmill during walking and running activities at different speeds. Spectral analysis was used to evaluate the frequency field of impact shock during walking and running activities. The effects of increasing impact shock levels are evaluated during walking and running in function of pedestrian's velocity. In addition, it is proposed the comparison between experimental data obtained by walking activity and experimental data obtained by running activity.

TheWilcoxon Rank-Sumnon-parametric test evaluates whether the two independent samples of walking and running are obtained from populations with an ordinal distribution. For this test we can adopt the following null hypothesis:

- $H_{0}$ : ratio between peak force and static weight during running exercise is greater than ratio between peak force and static weight during walking exercise.

This work can offer a comprehensive database on accelerations of pedestrian's foot. The key problem of this data set is 
that the variability of the Fourier coefficients and the activity rate harmonics can represent the variability of real walking or running between subsequent steps of the same pedestrian during walking or running activities. The mathematical approach and experimental investigation illustrate intra-subject variability of impact shock during walking and running activities.

The usefulness of this research is to explore the dependence of peak acceleration of foot on different pedestrian's velocity. Impact shock can provoke the injury of bone structure. Recommendations could be deducted with regard to the dose of impacts that can be beneficial or detrimental to human health.

\section{Materials and Methods}

It is proposed the mathematical model and the data processing, including sample collection and statistical tests are used for data analysis.

\subsection{Mathematical Model}

Under the assumption that an individual generates identical and therefore perfectly repeatable footfalls with frequency $f_{i}$, the accelereration $a(t)$ of the foot can be represented in time domain as a sum of Fourier harmonic components:

$$
a(t)=\sum_{i=1}^{n} \lambda_{i} \cdot \sin \left(2 \pi \cdot f_{i} \cdot t+\phi_{i}\right)
$$

where

- $i$ the order number of the harmonic;

- $n$ the total number of contributing;

- $\lambda_{i}$ the Fourier coefficient of the ith harmonic indicated as dynamic acceleration factor (DAF);

- $f_{i}$ the activity rate $(\mathrm{Hz})$ harmonics;

- $\phi_{i}$ the phase shift of the $i$-th harmonic (rad).

Fourier series is a quantitative approach to evaluate the movement or motion based on data acquired during walking and running analysis.

\subsection{Data processing and analysis}

This study adopts an instrumented treadmill measurement. Compared to a single force plate, treadmill technology allows analysis of stride-to-stride variations for many consecutive cycles over a long period of time. The test protocol usually requires 2 minutes of walking or running measurements. Typically between 100 and 120 steps can be recorded. This method provides for a more powerful statistical comparison.
The speeds were $1,2,3$ and $4 \mathrm{~m} / \mathrm{s}$ in walking activity and 4 , $5,6,7,8$ and $9 \mathrm{~m} / \mathrm{s}$ in running activity.

The experimental investigations was repeated three times for each subject in order to assess the repeatability of measurements. A warm-up of 10 minutes is enough for pretest habituation to treadmill walking and running activities. An accelerometer MPU6050 was connected to ATmega328P microntroller $16 \mathrm{MHz}$ to acquire experimental vertical accelerations on center gravity of foot (Figure 1).

Conventional statistical methods were used to calculate means and standard deviations (SD). Differences and comparisons were tested for significance with the Student's t-test and Wilcoxon Rank-Sumnon-parametric test. Values of DAF factors are presented as means and standard deviations. The probability level for statistical analysis was set at $p<0.05$.

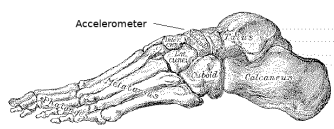

Figure 1. Side (medial) view of the bones of the left foot, showing the longitudinal arch

\section{Results}

The methods offers the variability of DAF values between subsequent steps of the same pedestrian during walking and running activities (Fig.2 and Fig.3). Statistical tests, used for data analysis, shows that the transition from walking activity to running activity provokes an increase of accelerations of foot.

The ratio between peak force of the foot on the ground and static weight, the walking velocity are important gait parameters to evaluate walking and running activities. Running activity provokes an increase of the ratio between peak force and static weight compared to walking activity (Fig.4). The accelerations of foot increases in the transition from walking activity to running activity (Fig.5). The ratio between peak force, caused by impact of foot on the ground, and static weight are within 2 standard deviations $\sigma$ of the mean $\mu$ (Table 1).

\section{Discussion}

In this study, the first key question concerns shock evaluation between foot and ground. Impacting on the ground, the foot causes a wave of shock throughout the skeletal system. Anatomical structures such as the heel pad, ligaments, articular cartilage and bone will passively attenuate the shock wave. Higher magnitude or more frequent impacts will place greater stress on these structures resulting in an increased risk of injury and degenerative disease. The musculo-skeletal system is also able to attenuate shock via active processes such as adjusting joint stiffness and manipulating kinematics to 
place body segments in positions that more readily attenuate shock. Subtalar pronation and increased knee flexion are two kinematic mechanisms that the body can use to attenuate shock.

If the impact of the foot on the ground causes deleterious effects on the human body, the runners can adopt a stride frequency that minimizes these effects [6]. The results of our study seem to support the notion that runners adopt a stride frequency that minimizes impact shock.

The bones of the foot are configured to form the arch but it is maintained under load principally by the plantar aponeuerosis which originates at the calcaneus and inserts into the proximal phalanges. This is an interesting aspect because of the structure of the arch offers energy savings during locomotion by storing some of the energy in the foot during ground contact.

There are very few muscles in the foot. Motion of the toes is controlled by muscles in the leg, the tendons of which are attached to the bones of the foot.

The number of dynamic variables, which can be described during walking and running activities, are the dynamic acceleration factors (DAFs), the activity rate $(\mathrm{Hz})$ harmonics $f_{i}$ and the phase shift $\phi_{i}$ of the $i$-th harmonic (rad). Transitioning from walking activity to running activity requires an increase of energy. In fact, if walking speed increases, DAF factors increase.

In this study the dynamic acceleration factors (DAFs) assume a relevant aspect during human locomotion. The analysis represents the variability of DAF values between subsequent steps of the same pedestrian during walking and running activities (Fig.2 and Fig.3).

The mathematical model represents a direct approach to measure the frequency content during the impact of the foot on the ground. Firstly, the approach filters the effect of contaminating noise on measured signals. The effects of this noise can be mitigated through the use of a digital filtering technique. Therefore, the noise frequencies do not overlap the signal frequencies. In addition, the experimental data are sampled at more than twice the highest frequency in the composite signal and noise to avoid aliasing. The accelerometer MPU6050 in an excellent sensor in order to estimate the impact conditions. However, the peak accelerations can be influenced by the soft tissue movement, relative to the bone.

In order to compare walking and running activities we evaluate the results by $t$ distribution and Wilcoxon RankSumnon-parametric test.

The $t$ distribution provides a good method to perform one sample tests on the mean when the population variance is not known. In addition, the $t$ distribution provides good results in many probability distributions and even when the sample is large or small. Therefore, it is applied Central Limit Theorem.

The $t$ distribution offers the result that $95 \%$ of ratio between peak force, caused by impact of foot on the ground, and static weight are within 2 standard deviations $\sigma$ of the mean $\mu$ (Table 1 ).

In addition, a comparison of statistical values between walking and running activities (Fig.5) shows that the transition
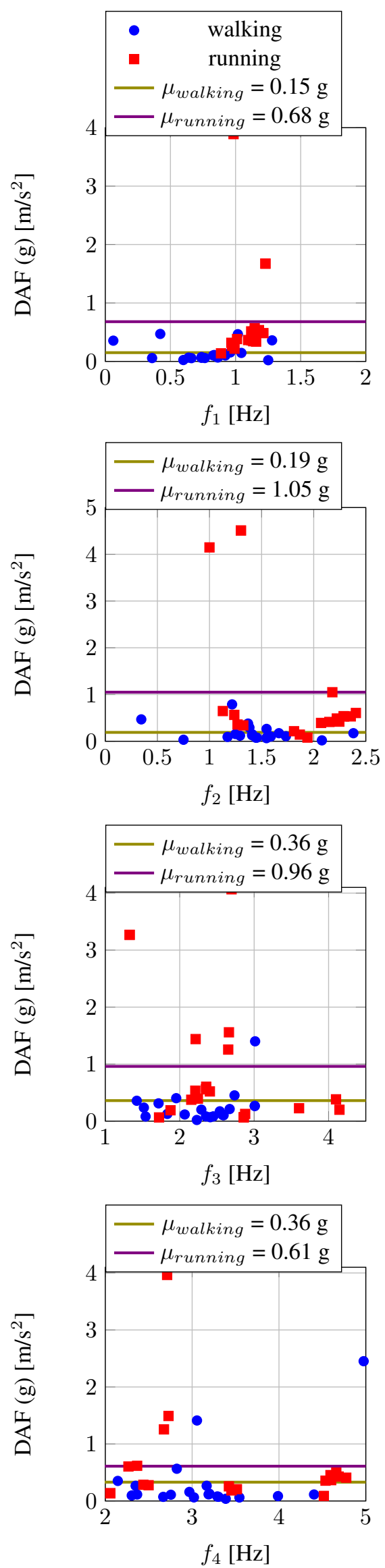

Figure 2. Comparison of DAF values obtained by walking and running activities for harmonics $1,2,3$ and 4 

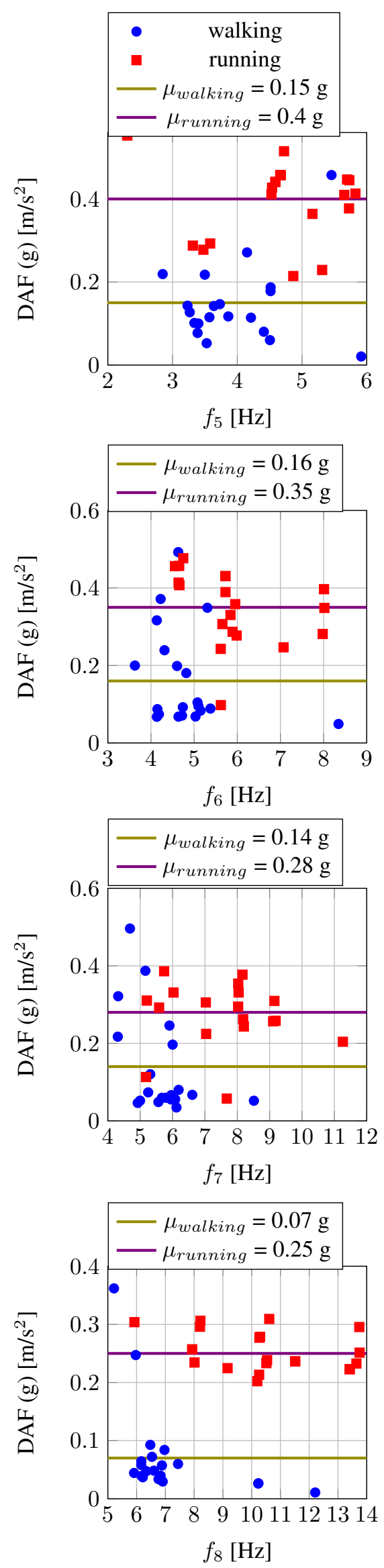

Figure 3. Comparison of DAF values obtained by walking and running activities for harmonics 5, 6, 7 and 8
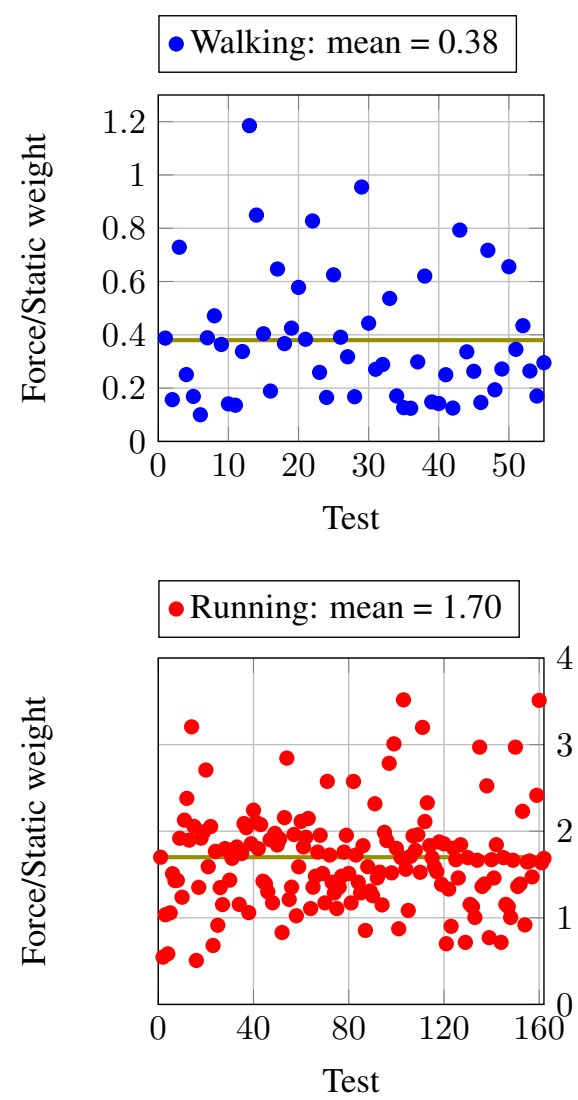

Figure 4. Force/Static weight during walking and running activities

from walking activity to running activity provokes an increase of accelerations of foot.

We compare the average rank $W$, obtained by walking and running activities, with the critical average rank $W_{c r i t}$, which can be found in the Wilcoxon Rank-Sum Table for $\alpha=.05$. In this study we obtain $W<W_{\text {crit }}$. Therefore we can accept the null hypothesis. It means that there is a significant difference between the effectiveness of the walking activity and the running activity. The ratio between peak force and static weight during running activity is always greater than ratio between peak force and static weight during walking activity (Fig.4).

The walking activity, respect to running activity, causes lower impulses during each foot contact, but the walking provokes a higher number of foot contacts per unit of time. For against, the running activity, respect to walking activity, causes higher impulses during each foot contact.

If the pedestrian increases the walking or running velocity, the peak amplitude of the vertical reaction force in walking and running activities increases respect to static weight.

The ratio between peak force and static weight is about 1.0 at walking velocity $1 \mathrm{~m} / \mathrm{s}$. At higher walking speeds $4 \mathrm{~m} / \mathrm{s}$, the ratio between peak force and static weight increases from 1 to 2.0 times respect to body weight (Fig.6).

The ratio between peak force and static weight is about 2.0 at running velocity $4 \mathrm{~m} / \mathrm{s}$. At higher running speeds 9 $\mathrm{m} / \mathrm{s}$, peak forces increases and it becomes approximately 3.0 times body weight (Fig.6). 
We can affirm that walking velocity and peak force are good gait parameters to evaluate walking and running activities. The mean ratio between peak force $F$ and static weight $W$ can be considered function of the walking frequency. We noted a considerable intra-subject variation of this mean ratio for the velocity from $1 \mathrm{~m} / \mathrm{s}$ to $9 \mathrm{~m} / \mathrm{s}$. Due to the intra-subject variability, the walking force parameters (Fig.6) are not constants but fluctuate with time and change from one stride to the next stride. The ratio between the peak force and static weight increases from $1 \mathrm{~m} / \mathrm{s}$ to $6 \mathrm{~m} / \mathrm{s}$ and assumes a flat trend from $6 \mathrm{~m} / \mathrm{s}$ to $9 \mathrm{~m} / \mathrm{s}$.

Similarly, Wheeler noted considerable inter-subject variation of the ratio between the peak force and static weight [8]. Wheeler examined the frequency field from $1 \mathrm{step} / \mathrm{s}$ to 5 step/s.

\section{Force/Static weight}

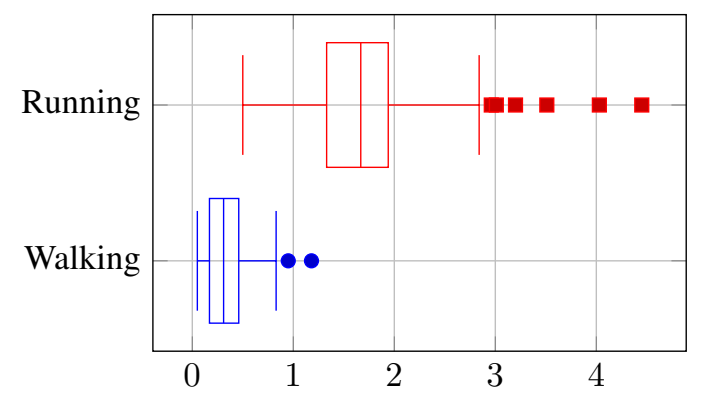

Figure 5. A comparison of statistical values between walking and running activities

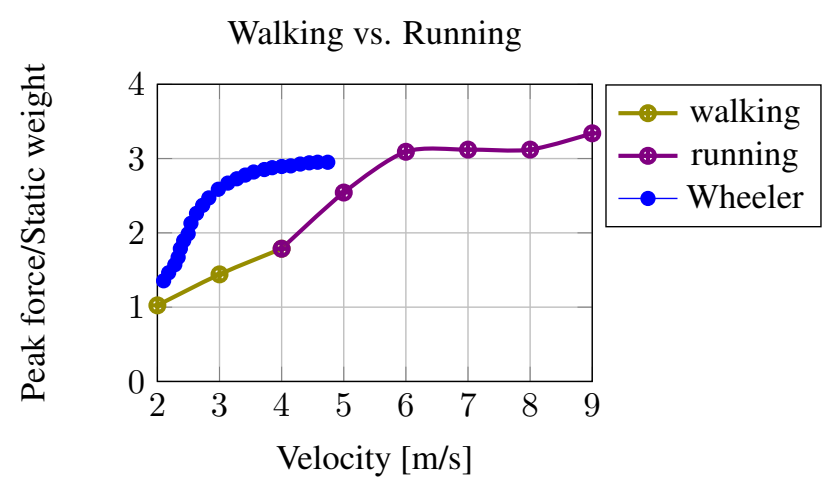

Figure 6. Dependence of peak force on different pacing rates

\begin{tabular}{cccccc}
\hline Activity & $\mu$ & $\sigma$ & $t_{\text {stat }}$ & $t_{\text {crit }}$ & $p$ \\
\hline Walking & 0.38 & 0.24 & 11.55 & 1.67 & $<0.05$ \\
\hline Running & 1.7 & 0.65 & 33.22 & 1.65 & $<0.05$ \\
\hline
\end{tabular}

Table 1. $\mathrm{t}$ Test for walking and running

\section{Conclusions}

Shape and amplitude of the vertical accelerations are important parameters to evaluate the impact of the foot on the ground. Marked differences in vertical accelerations are illustrated between walking and running activities. The differences are a consequence of movement strategies between the two major forms of human progression.

Future aspect of this research is to develop the analysis of transmissibility for the human spine during walking and running activities.

\section{REFERENCES}

[1] A. H. Gruber, K. A. Boyer, T. R. Derrick, J. Hamill, Impact shock frequency components and attenuation in rearfoot and forefoot running, Journal of Sport and Health Science, 2014, Vol.3, 1-14.

[2] C. L Vaughan, B. L. Davis, J. C. O'Connor, Dynamics of Human Gait, 1999, Kiboho Publishers, ISBN: 0-620-23560-8, South Africa.

[3] Y. Matsumoto, M. J. Griffin, Effect of muscle tension on non-linearities in the apparent masses of seated subjects exposed to vertical to vertical whole-body vibration, Journal of Sound and Vibration, 2002, Vol. 253, 77-92.

[4] J. Lim, M. A. Busa, R. E. A. van Hemmerik, J. Hamill, Adaptive changes in running kinematics as a function of head stability demands and their effect on shock transmission, Journal of Biomechanics, 2017, Vol. 52, 122-129.

[5] S. P. Nigam, M. Malik, A Study on a Vibratory Model of a Human Body, Journal of Biomechanical Engineering, 1987, Vol. 109, 148-153.

[6] A. I. King, The Biomechanics of Impact Injury, Springer, Journal of Biomechanical Engineering, 2018, Switzerland, ISBN: 978-3-319-49790-7, DOI: 10.1007/978-3-319-49792-1.

[7] M. R. Shorten, D. S. Winslow, Spectral Analysis of impact Shock During Running, International Journal of Sport Biomechanics, 1992, Vol.8, 288-304.

[8] J.E. Wheeler, Prediction and control of pedestrian induced vibration in footbridges, Journal of the Structural Division ASCE, 1982, Vol.108, 2045-2065. 